\title{
LA CADENA TECNOLÓGICA OPERATIVA COMO UNA HERRAMIENTA TEÓRICA Y METODOLÓGICA. UNA PERSPECTIVA DESDE LOS PLANTEAMIENTOS DE LA ARQUEOLOGÍA DEL PAISAJE
}

\author{
Por \\ ISABEL COBAS-FERNÁNDEZ \\ $M^{a}$ PILAR PRIETO MARTÍNEZ
}

\section{RESUMEN}

Centraremos nuestra comunicación en dos aspectos: el enfoque particular que hemos dado a la Cadena Técnica Operativa en el marco de la Arqueología del Paisaje para aplicarlo al estudio de la cerámica prehistórica y protohistórica del NW de la Península Ibérica y los problemas con los que nos hemos encontrado para la aplicación práctica de esta herramienta en dicho contexto.

Por lo que respecta al primer punto, creemos que la mayor novedad reside en que la CTO no se considera como un útil meramente descriptivo de hechos tecnológicos sino que se intenta relacionar estos hechos con el contexto social en el que se producen. Por esta razón hemos dividido la CTO en tres aspectos profundamente imbricados: los aspectos técnicos, considerados en la cadena técnica, los aspectos sociales, comprendidos en la cadena conceptual y, finalmente, el resultado de esos dos procesos, el producto final, que constituye el último eslabón de dicha cadena.

Por lo que hace al segundo punto, existen grandes problemas para conocer los aspectos tecnológicos, puesto que se necesita del concurso de 
técnicas auxiliares no siempre disponibles, pero sobre todo resulta difícil conocer la dimensión imaginaria al aproximarnos a ella únicamente a través de su plasmación material.

\title{
PALABRAS CLAVE
}

Cadena Operativa, Cadena Tecnológica Operativa, Cerámica, Análisis Formal, Estilo, Patrones de Regularidad Formal, Arqueología del Paisaje.

\begin{abstract}
We will focus on two main topics: first, the special approach to the Technological Chain (TC) that we have used to study NW Iberian protohistoric and prehistoric pottery from a landscape archaeology viewpoint; and second, the specific problems that we found while trying to perform such work.

Regarding the first point, we believe that the most innovative issue is that the TC is not only seen as a mere descriptive tool for technological facts, but we have tried to relate such facts with the social context in which they occur. As a result of it, we have divided the TC into three deeply intertwined aspects: the technical aspects or technological chain, the social aspects or conceptual chain, and the final result of those processes, the final product, which comprises the last link in the chain.

Concerning the second point, we have found important obstacles in trying to get to the technological aspects, because the necessary auxiliary techniques are not always readily available. Also, gaining knowledge of the imaginary dimension is even more difficult, as we approach it solely from its material demonstration.
\end{abstract}

\section{KEYWORDS}

Operative Sequence, Operational Technological Chain (OTC), Ceramics, Formal Analysis, Style, Patterns of Formal Regularity, Landscape Archaeology. 


\section{INTRODUCCIÓN}

El planteamiento teórico del cual partimos es el de la Arqueología del Paisaje $^{1}$, según el cual las entidades arqueológicas no existen aisladas sino que están determinadas por todas las instancias sociales que construyeron este registro. Esto quiere decir que las entidades arqueológicas son entidades espaciales, formas producidas por la acción social, relacionadas con un contexto socio-cultural y comprensibles dentro de él. En coherencia con esto, la cultura material se entiende como objetificación del ser social, tal y como proponen Shanks y Tilley (1987:130, citado por Criado 1993b: 41) ${ }^{2}$, es decir, como producto de una determinada sociedad que responde a unas pautas culturales concretas, por lo cual la cultura material «debe ser interpretada en términos de coherencia con el sistema cultural total» (Rivera 1990: 24), ya que todo el proceso seguido en la elaboración de la misma, desde la propia obtención de la materia prima hasta la consecución del producto final, está condicionado, intencional o no intencionalmente, por las circunstancias sociales en las que ésta se inscribe, convirtiéndose de este modo en reflejo y parte activa del contexto social en el que éste fue producido ${ }^{3}$.

El modo de estudio que proponemos es el que hemos llamado Análisis Formal $^{4}$ (Fig. 1), consistente en la deconstrucción de las características de

\footnotetext{
${ }^{1}$ Los principios básicos de este enfoque teórico han sido expuestos por Felipe Criado Boado en diversos artículos (p.ej.: 1993a, 1993b, 1999).

${ }^{2}$ Lo que se propone en definitiva es un estudio de la cultura material como «objeto situado en el mundo social como objeto» (Boast 1995).

${ }^{3}$ Lo que aquí presentamos es únicamente un pequeño resumen de una línea de investigación sobre cultura material cerámica prehistórica y protohistórica que se ha ido desarrollando desde 1993 hasta la actualidad dentro del Gabinete de Cultura Material perteneciente al Laboratorio de Arqueología y Formas Culturales de la Universidad de Santiago de Compostela dirigido por Felipe Criado. Los trabajos que se pueden consultar acerca de esta línea de investigación son Cobas 1995 y 1997, Cobas y Prieto 1997, 1998a, 1998 b y 2002, Prieto $1993,1995,1996,1998,1999,2002 a, 2002$ b y 2002 c.

${ }^{4}$ En el estudio de los mitos realizado desde la Antropología Estructural se dice que una de las finalidades de la descripción es «identificar e inventariar tipos, analizar sus partes constitutivas, establecer entre ellos correlaciones. Sin este trabajo preliminar (...) el método comparativo (...) corre peligro de fracasar: o bien los datos que uno se propone comparar son tan próximos geográfica o históricamente, que jamás se tiene la seguridad de hallarse ante fenómenos distintos, o bien son demasiado heterogéneos y la confronta-
} 


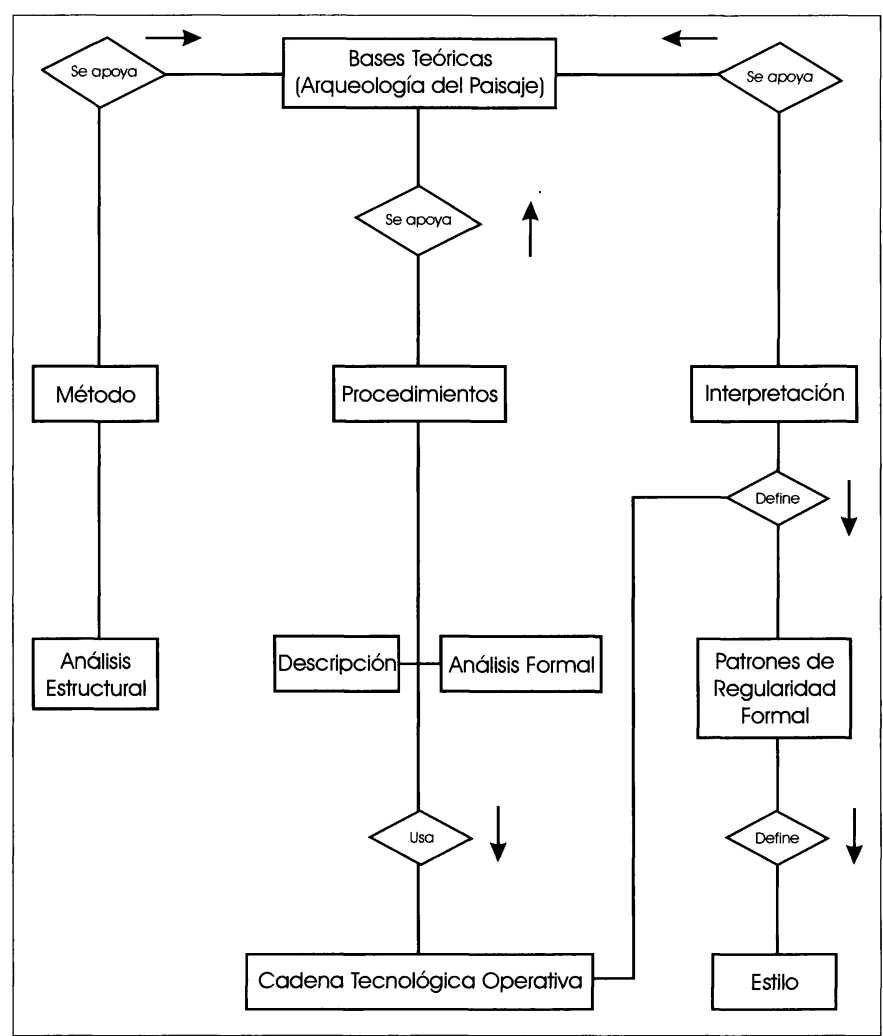

Fig. 1. Fases del proceso de trabajo.

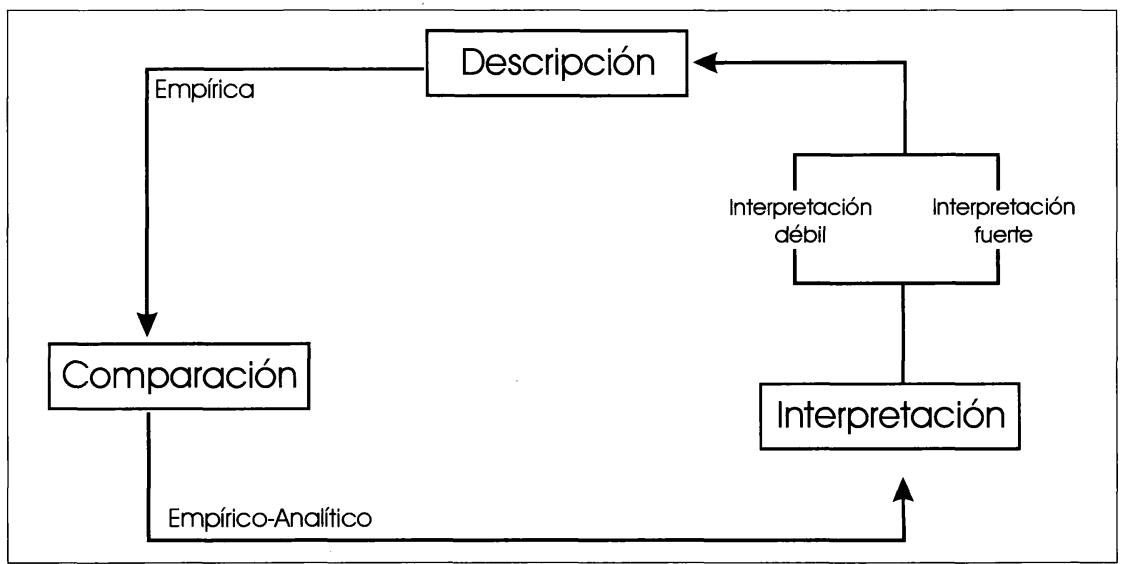

Fig. 2. Esquema del proceso de trabajo.

«CUADERNOS DE ESTUDIOS GALLEGOS», Tomo XLVIII, Fascículo 114, Santiago 2001. 
los objetos mediante un proceso descriptivo y la posterior reconstrucción de las mismas a través de un proceso interpretativo.

Este estudio se realiza a través de tres fases sucesivas que van abordando progresivamente mayores grados de subjetividad e interpretación: En primer lugar se lleva a cabo la descripción de los objetos, consistente en un proceso de carácter previo sobre el que se asienta todo el desarrollo posterior del trabajo, en el que se intentan reconocer las características físicas de los objetos ${ }^{5}$. A continuación se realiza la clasificación de los objetos, esto es, la reordenación de las relaciones formales (Criado 1993b: 53) y el reconocimiento de los rasgos formales que permiten definir tendencias y variantes, o lo que es lo mismo, tipos y subtipos dentro del conjunto material estudiado y, por último, se aborda la interpretación, en la que se lleva a cabo la elaboración de los datos obtenidos en los pasos anteriores, intentando relacionarlos con un patrón de racionalidad común, mediante la contrastación de los datos de la cultura material con otros relativos a esa cultura y, en los casos en los que sea posible, mediante la relación de esos datos con una teoría antropológica (Fig. 2). En resumen, el objetivo del estudio es reconstruir los patrones de regularidad existentes entre los objetos con el objetivo de caracterizar estilos ${ }^{6}$.

\section{LA CADENA OPERATIVA}

En relación con lo expuesto anteriormente, creemos que la cadena operativa es el instrumento que reúne de modo más manifiesto todos los principios sobre los cuales se fundamenta nuestra propuesta para el estudio del material, ya que en ella se conjugan aspectos de carácter descrip-

ción se vuelve ilegítima porque aproxima cosas que no son comparables» (Lévi-Strauss 1987: 29), nosotros compartimos esta definición y creemos que es perfectamente aplicable a los estudios cerámicos.

${ }^{5}$ El desarrollo del trabajo con el material en tres fases es una propuesta común en la mayoría de los autores: Rivera (1990), Hodder (1988), Bate (1978) Delgado (1989).

${ }^{6}$ Entendemos el concepto de Estilo en un sentido amplio, «como uno de los mecanismos del discurso de poder según éste se refleja en los productos formales de la sociedad, esto es, el estilo sería la formalización externa del poder, entendiendo poder en sentido foucaultiano» (Prieto 1999: 75) (Fig. 4).

«CUADERNOS DE ESTUDIOS GALLEGOS», Tomo XLVIII, Fascículo 114, Santiago 2001. 
tivo, analítico e interpretativo para plasmar gráficamente los patrones formales de regularidad y las diferencias en la cultura material a lo largo del proceso tecnológico completo, en la medida que lo permiten los restos arqueológicos, para poder caracterizar el contexto de las sociedades primitivas pasadas, a través de la comparación en múltiples niveles de complejidad. Descriptivo, toda vez que se realiza a partir de los datos obtenidos en la primera parte del trabajo. Analítico por cuanto tiene en cuenta las distintas fases de elaboración del material en un conjunto material dado. Interpretativo, puesto que constituye un intento hipotético de acceder no únicamente al modo cómo se realizó el cacharro, si no a los condicionantes y circunstancias que se ocultan bajo ese proceso de fabricación ${ }^{7}$.

\subsection{Revisión historiográfica del uso de la cadena técnica}

Al hablar de cadena técnica inevitablemente debemos hacer referencia al origen de este concepto en el contexto de estudio de la industria lítica, a pesar de que para su aplicación al estudio del material cerámico no se ha hecho una transposición directa del término tal y como éste se utilizó en los primeros momentos, como útil descriptivo, desde la Arqueología Histórico-Cultural (Léroi-Gourhan 1965) y posteriormente también como útil analítico, sobre todo desde una perspectiva funcionalista (Binford 1989), sino más bien en relación con los desarrollos que este concepto ha adquirido en los últimos tiempos, básicamente desde la Antropología de la Tecnología (Lemonnier 1986, 1991a, 1991b, Gosselain 1992), en los que se explora su potencial interpretativo ${ }^{8}$.

\subsection{El concepto de cadena técnica desde la Arqueología del Paisaje}

La principal diferencia que creemos que se incorpora desde la Arqueología del Paisaje en la aplicación del concepto de cadena técnica a los estudios cerámicos radica en que no sólo se pretende reconstruir una se-

\footnotetext{
${ }^{7}$ Estos tres principios se oponen estructuralmente a los conceptos de tipo y tipología, nociones que han marcado desde los inicios de la literatura arqueológica los estudios de cultura material utilizando la tipología como instrumento metodológico clave y la definiciones del tipo objetivo final del estudio (Fig. 3).

${ }^{8}$ Ver un estudio historiográfico reciente sobre este tema en Martinón (2002).
} 


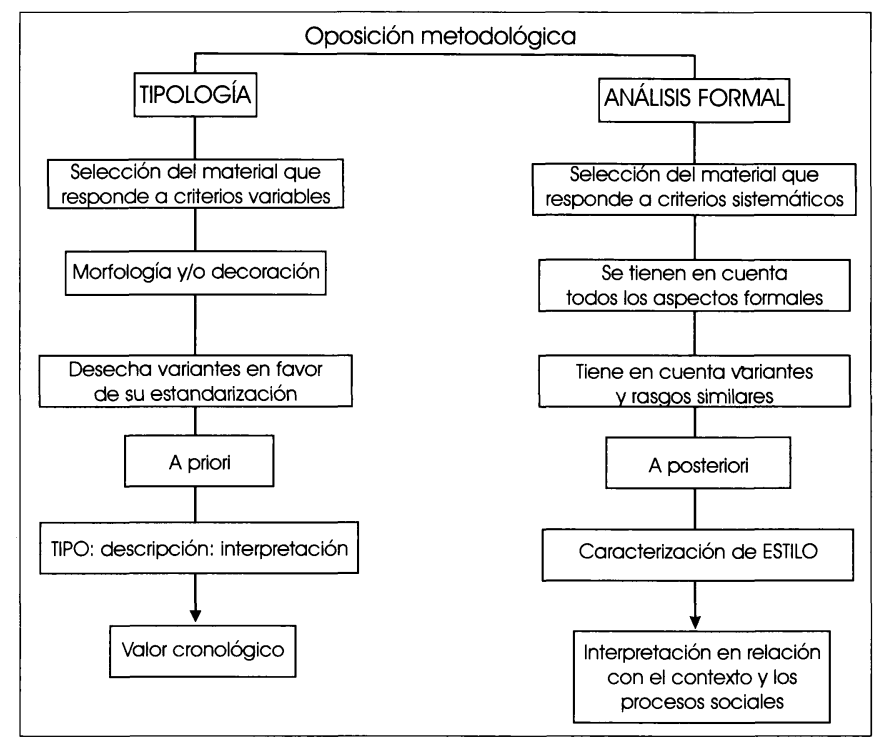

Fig. 3. Esquema explicativo de dos metodologías aplicadas al estudio de la cerámica.

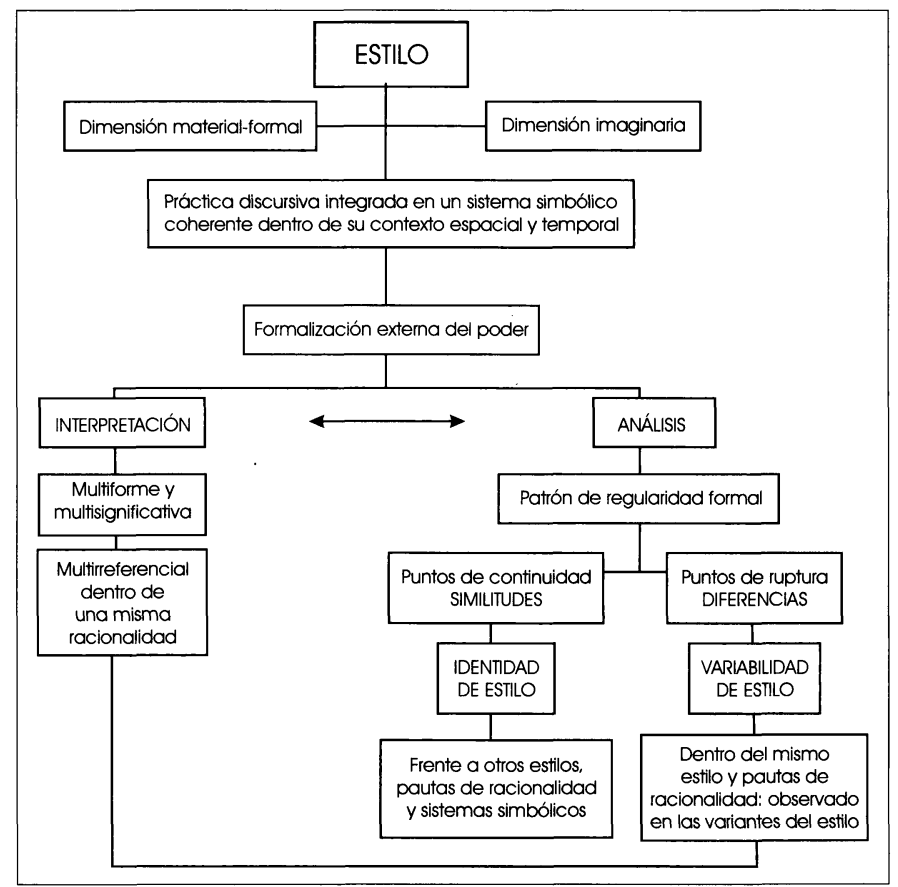

Fig. 4. Dimensiones significativas del Estilo desde la Arqueología del Paisaje.

«CUADERNOS DE ESTUDIOS GALLEGOS», Tomo XLVIII, Fascículo 114, Santiago 2001. 
cuencia física de gestos seguidos hasta la obtención de un producto acabado (Julieu 1992: 176-79) sino que se presta especial importancia a los aspectos conceptuales implicados en esa secuencia de gestos y al modo como el producto acabado se relaciona con el contexto social. Por esta razón, en lugar de utilizar el término cadena operativa, nos inclinamos por utilizar el concepto cadena tecnológica-operativa, pues creemos que el término tecnología, entendido como «un saber que permite hacer cosas» (Criado 1996: 26), se ajusta mejor a la realidad estudiada en la que no sólo es importante la capacidad física de llevar a cabo un producto sino la posesión de un conocimiento simbólico acorde con el contexto social en el que este producto se realiza.

Entendemos el concepto de cadena tecnológica-operativa como un útil analítico que permite la descripción ordenada de las instancias y circunstancias que determinan el proceso de elaboración de los elementos cerámicos. Estas instancias se pueden ordenar esencialmente en tres grupos: los aspectos técnicos en sentido estricto, considerados en lo que podría denominarse propiamente la cadena técnica, los aspectos referidos a las instancias sociales en las que se combina tanto el grupo social como el individuo y el contexto histórico, que denominamos cadena conceptual $\mathrm{y}$, finalmente, el resultado de esos dos procesos, definido como producto final. No obstante, se trata de una división únicamente desde el punto de vista práctico, ya que todos ellos se encuentran interrelacionados. A continuación explicamos con mayor detalle cada uno de estos aspectos (Fig. 5).

Cadena técnica. Dentro de ella realizamos una diferenciación entre las fases de elaboración, entendiendo por ello la sucesión de ciclos que se suceden en la producción de un objeto cerámico, y los procesos de elaboración, referidos a los modos de actuación y trabajo o tareas concretas en cada una de esas fases, como entidades menores que posibilitan el desarrollo de cada uno de los ciclos.

Cadena conceptual. Se tienen en cuenta los condicionantes de orden económico, territorial, social, imaginario, que confluyen y señalan todo el proceso de fabricación de la cadena técnica. La manera de elaborar un cacharro, desde la primera hasta la última fase, forma parte de una intención social, integrada en una práctica discursiva concreta, que 


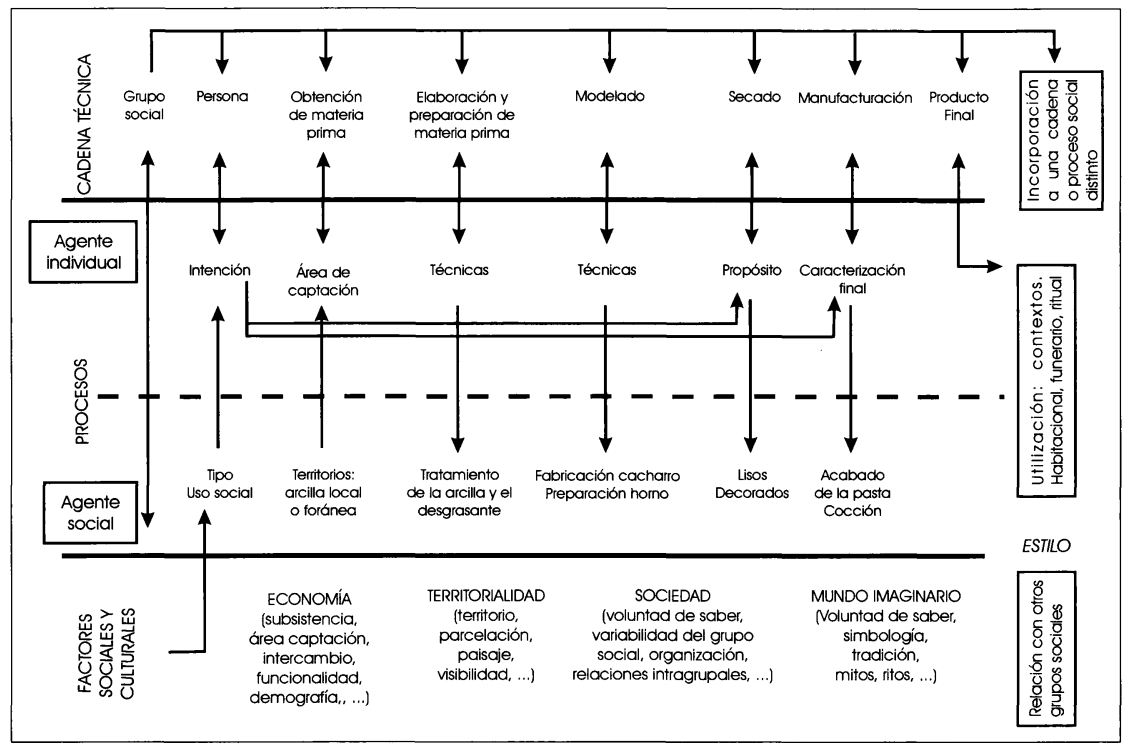

Fig. 5. La Cadena Tecnológica Operativa.

está relacionada con la manera de hacer de un grupo social dado, y que por encima de diferencias o matices en muchas de las fases de producción, permite reconocerlo dentro de un marco social más amplio que indicará la identidad de estilo. Es la voluntad de saber-poder de cada grupo el que determina las diferentes prácticas discursivas que se desarrollan en su propio seno ${ }^{9} \mathrm{y}$, por tanto, la que marcará el tipo de uso social al que irá destinado el producto. Por lo tanto, no consideramos que sea posible establecer cadenas tecnológico-operativas válidas universalmente tomando como única referencia los aspectos técnicos ya que, tal y como señala Perlés (1991: 9), la cadena tecnológica-operativa «no es un sistema inferencial desde lo concreto hasta lo abstracto del esquema conceptual y de conocimiento, sino que se inscribe también en

${ }^{9}$ Siguiendo la idea de voluntad de saber-poder de Foucault (1984). Creemos que esta noción se puede aplicar, aunque por supuesto no de forma directa, a las sociedades prehistóricas (Criado Boado, 1989: 78 y Méndez Fernández, 1994: 79); por lo tanto, el concepto de poder no debe entenderse en un sentido estricto ya que Foucault lo aplica a las sociedades modernas occidentales. Las hipótesis que plantea en torno a la noción de poder puede verse en Poderes y estrategias (Foucault, 1980: 170-1 y 1981: 82).

«CUADERNOS DE ESTUDIOS GALLEGOS», Tomo XLVIII, Fascículo 114, Santiago 2001. 
un tiempo y en un espacio». No existe una relación directa entre sociedad y cultura material tomados como conceptos generales e inmutables, si no que existe un elemento intermedio o tercer factor (Criado 1984$85)$, constituido por el conjunto histórico individual, que lo condiciona y hace característico.

El resultado de todo este proceso será el producto final. Con ello volvemos a la definición que dábamos al principio de este texto, puesto que entendemos el producto como una forma, plasmación formal o conjunto de características formales adquiridas como resultado de la «mediación gestual del hombre sobre la materia» (Delgado 1988) determinada por instancias sociales, que debido a esa determinación social se inscribe dentro de un patrón de regularidad formal estipulado coherente con otros códigos y, en última instancia, reflejo del patrón de racionalidad en el que se inscribe. De este modo, la cultura material adquiere un valor no sólo funcional (real) sino también simbólico (imaginario), como elemento a través del cual se reflejan las pautas culturales de la sociedad en la que se produce, sirviendo así como transmisor de su sistema de valores y creencias, «aunque eso no implique que en la realidad cotidiana actúen siempre de acuerdo a esos valores» (Rivera 1990: 24 y 25). Este producto final entrará a formar parte de la cadena o proceso social, que constituirá el contexto original de la pieza ${ }^{10}$. Posteriormente, sobre el producto final y una vez que éste ha llegado al final de su etapa útil, se sucederán una serie de procesos postdeposicionales ${ }^{11} \mathrm{y}$, finalmente, irrumpirá el arqueólogo que intentará acercarse al conocimiento de todo ello desde un contexto radicalmente diferente ${ }^{12}$.

\footnotetext{
${ }^{10}$ Lo que Boast denomina la biografia útil de la cerámica (Boast 1995: 70).

${ }^{11}$ Denominado por Boast biografia de deposición (Boast 1995: 70).

${ }^{12}$ El mayor problema en el proceso de reconstrucción del significado de la pieza es que a la propia subjetividad del arqueólogo se suma el inconveniente, no siempre tenido suficientemente en cuenta, de que dicha reconstrucción ha de hacerse contando únicamente con la biografia de deposición. Por ello, son importantes los trabajos que están empezando a desarrollarse sobre el modo como los materiales se integran en el registro arqueológico y la relación entre su estado actual y sus posibles características originales (Evans y Millet 1992, Hill 1995b, Maltby 1985) que, aunque orientadas bajo un marco teórico diferente, siguen líneas ya comenzadas anteriormente (Schiffer 1976), y llaman la atención sobre los peligros derivados de la realización de una lectura directa sobre el material cerámico.
} 
Bajo este enfoque, el planteamiento de trabajo que realizamos para la cerámica intenta evitar tanto la concepción del cacharro únicamente como objeto museable, como el tratamiento de la cerámica «como personas» (Boast 1995) $)^{13}$. Antes bien, estudiamos la cultura material como un código producido por una cultura, que refleja mediante sus características formales, y por tanto visibles y susceptibles de ser descritas, el patrón de racionalidad subyacente a la misma.

\section{POSIBILIDADES Y LIMITACIONES DEL USO DE LA CTO}

El uso de la cadena tecnológica-operativa ofrece importantes novedades en el estudio de la cultura material cerámica y sobre todo en la definición de los estilos, ya que, frente al concepto de estilo habitual, basado únicamente en los aspectos más materiales, evidentes y susceptibles a los cambios (Lemonnier 1986), esto es la morfología y la decoración, representados en el objeto acabado ${ }^{14}$, se estudia toda la variedad de elecciones a lo largo de la cadena tecnológica-operativa que se producen dentro de una serie de opciones igualmente viables, destacando el rol activo del objeto en las sociedades pretéritas y la elección cultural $^{15}$ variable para cada sociedad (elecciones tecnológicas socialmente pertinentes según Lemonnier 1986: 153) ${ }^{16}$. Al incorporar el concepto de cadena tecnológica-operativa se incorpora el aspecto tecnológico como un nuevo parámetro de estilo haciendo que al seguir los procesos tecnológicos podamos acce-

\footnotetext{
${ }^{13}$ Encontramos una idea similar en otros autores como Gruber 1986 y Sackett 1983, que intentan evitar la identificación directa entre cerámica y culturas que prevalecen en los estudios de tipo evolucionista o difusionista.

${ }^{14} \mathrm{Tal}$ y como se refleja en la definición de estilo como el «conocimiento formal de los modos particulares en los que los diferentes artefactos presentan similitudes entre ellos» (Davis 1986).

${ }^{15}$ Estas elecciones se pueden ver en dos niveles: un nivel particular donde se pueden obtener diferentes combinaciones para la elaboración de un mismo código de cultura material, o a un nivel particular, donde se pueden contrastar elecciones y combinaciones para la producción de objetos de cultura material de diversa índole.

${ }^{16}$ Este concepto presenta rasgos en común con la propuesta de Gosselain (1992), que toma como punto de partida la definición de estilo de Sackett (1990) y la reconvierte a través de su inmersión en la línea de trabajo de la Antropología de la Tecnología desarrollada sobre todo por Pierre Lemonnier (p.ej. 1983, 1986, 1991a, 1991b)
} 
der a criterios menos susceptibles a los cambios y que ofrecen una idea más precisa de las sociedades del pasado (Gosselain 1992).

El empleo del concepto de cadena operativa tiene varias implicaciones tanto metodológicas como interpretativas. La más inmediata, respondiendo a una orientación metodológica, supone ampliar los ámbitos de estudio ya que existen fases dentro de la cadena operativa que no pueden ser reconstruidos mediante la arqueología, especialmente los referidos a los factores sociales que influyen en ella, para lo cual se necesitaría el recurso a la etnología mediante analogías débiles, o lo referente a los procesos tecnológicos, para cuya identificación no basta con una observación visual sino que es preciso realizar análisis físico-químicos. Desde el punto de vista interpretativo. Debemos ser conscientes de que, mientras es posible tener acceso a la cadena técnica y los procesos que se desarrollan para la realización de un producto a través del registro arqueológico, la consideración de los factores culturales nos llevan a un terreno más hipotético, ya que los condicionantes que marcan el proceso de fabricación (tales como la tradición oral o la mitología de esas sociedades) no se pueden documentar de forma directa, y casi ni siquiera de forma indirecta, en el registro arqueológico ${ }^{17}$.

A partir de la identificación de elecciones culturales no llegamos directamente a los esquemas conceptuales subyacentes a ellas ya que las características de la cultura material deberían contrastarse con las implicaciones sobre la naturaleza y el funcionamiento de una determinada sociedad para, a través de esa contrastación, acercarnos al patrón de racionalidad que vincula ambas esferas (cfr.: Rivera 1990). El problema reside en la imposibilidad de conocer los esquemas sociales al tratarse de sociedades desaparecidas y dotadas de nociones organizativas diferentes a las nuestras, por lo que las dificultades ya existentes para captar todos los matices de la construcción de un cacharro en el marco de las socieda-

${ }^{17}$ La mitología legitima el origen de operaciones técnicas particulares (Lemonnier, 1993: 19), por ejemplo en África entre los Bafia son las mujeres las que fabrican cerámica pero la invención se le atribuye a los hombres (Gosselain, 1992), también existen tabúes en relación con algunas de las secuencias tecnológicas, quizás las menos controladas por el propio alfarero a nivel tecnológico. En América el arte de la alfarería es también objeto de cuidados, de prescripciones y de prohibiciones múltiples (Lévi-Strauss, 1986).

«CUADERNOS DE ESTUDIOS GALLEGOS», Tomo XLVIII, Fascículo 114, Santiago 2001. 
des actuales (García Alén 1984: 58) se multiplican cuando intentamos acceder a la comprensión del material producido por una sociedad desaparecida y diferente a la nuestra ${ }^{18}$ ya que, desconocemos los significados subyacentes bajo la actuación sobre el material y estos todavía quedan más ocultos cuando sobre esas sociedades intentamos aplicar directamente nuestros propios patrones de racionalidad distorsionando su significado original ${ }^{19}$.

En resumen, el tipo de análisis que intentamos plantear presenta la contrariedad de añadir un componente de subjetividad en el estudio cerámico pero, una vez aceptado que dicho componente es inherente a la práctica arqueológica, lo que intentamos es evitar caer en una subjetividad incontrolada y abusiva, sino crear una metodología y un enfoque teórico determinados, y bajo el supuesto de que los diferentes códigos producidos por una misma cultura como respuesta a unas mismas pautas culturales pueden responder a patrones de regularidad similares (LéviStrauss 1986: 237 y ss.), intentar un aproximación a la realidad pretérita a través de su cultura material y concretamente el material cerámico. Bajo estos fundamentos se defiende el recurso a la línea estructural aplicada al estudio de sociedades actuales ya que, como señala Criado (1993b: 53), aunque el hecho de no trabajar con sociedades actuales y no poder acceder a su lenguaje sea la causa fundamental de que en arqueología no pueda realizarse un análisis estructural tal y como lo plantea Lévi-Strauss, esto no impide que existan postulados y formas de proceder que pueden ser muy útiles desde el punto de vista metodológico.

\section{BIBLIOGRAFÍA}

BATE, L.F. 1978. Formación económico-social y cultura. México: Ediciones Cultura Popular.

\footnotetext{
${ }^{18}$ Ejemplos de estudio de la cerámica en sociedades actuales como complemento de las sociedades pretéritas son los realizados por Varela (1990) o Delneuf (1991).

${ }^{19}$ Se está tomando cada vez mayor conciencia de los problemas derivados de la manipulación del registro arqueológico (p.ej.: Hill y Cumberpath 1993, Hill 1989, 1993, 1994, Le Roux y Gouyonvarc'h 1991).
} 
BINFORD, L. R.. 1989. En busca del pasado. Barcelona: Crítica (1 ${ }^{\text {st }} \mathrm{ed}$. 1988: In pursuit of the past. Decoding the archaeological record. London.).

BOAST, R. 1995. Fine pots, pure pots, beakers pots. In Kinnes and Varndell (eds.), 'Unbaked Urns of Rudely Shape'. Essays on British and Irish Pottery for Ian Longworth. Oxbow Monogrpah 55: 69-80.

COBAS FERNÁNDEZ, Mª I. 1995. Bases metodológicas para la descripción y estudio formal de la cerámica del yacimiento de Alto do Castro (Cuntis, Pontevedra). (Trabajo de Investigación del Tercer Ciclo). Laboratorio de Arqueoloxía e Formas Culturais, Departamento de Historia I, Facultad de Xeografía e Historia, USC. Santiago de Compostela. Inédito.

COBAS FERNÁNDEZ, Ma ${ }^{\mathrm{a}}$ I. 1997. Estudio de la cerámica castreña del yacimiento de Alto do Castro (Cuntis, Pontevedra). (Tesis de Licenciatura). Laboratorio de Arqueoloxía e Formas Culturais, Departamento de Historia I, Facultad de Xeografía e Historia, USC. Santiago de Compostela. Inédito.

COBAS FERNÁNDEZ, Mª I.; PRIETO MARTÍNEZ, Mª P. 1998a. Defining social and symbolic changes from the Bronze Age to Iron Age through the Operational Chains in NW Iberian pottery. En S. Milliken \& M. Vidale (eds.). Craft Specialization: Operational Sequences and Beyond. Papers from the EAA Third Annual Meeting at Ravenna 1997. Volume IV. BAR International Series 720: 95-106. Oxford.

COBAS FERNÁNDEZ, Mª I.; PRIETO MARTÍNEZ, Mª P. 1998b. Regularidades espaciales en la cultura material: la cerámica de la Edad del Bronce y la Edad del Hierro en Galicia. Gallaecia, 17: 151-75.

COBAS FERNÁNDEZ, Ma I.; PRIETO MARTÍNEZ, Mª P. 2002 (e.p.). The technological chain as a methodological and theoretical tool from archaeology. XIVth Congress of the U.I.S.P.P. Liège, Belgique, 2-8 septiembre 2001. Oxford: Archaeopress. 
CRIADO BOADO, F. 1984-85. «El tercer factor» o la lógica oculta del emplazamiento de túmulos megalíticos. Cuadernos de Estudios Gallegos, 35: 7-18. Santiago de Compostela.

CRIADO BOADO, F. 1993a. Límites y posibilidades de la Arqueología del Paisaje. SPAL 2: 9-56. Sevilla.

CRIADO BOADO, F. 1993b. Visibilidad e interpretación del registro arqueológico. Trabajos de Prehistoria, 50: 39-56. Madrid.

CRIADO BOADO, F. 1996. El futuro de la arqueología. ¿la arqueología del futuro?. Trabajos de Prehistoria, 53 (1): 15-35. Madrid.

CRIADO BOADO, F. 1999. Hacia una arqueología de los paisajes imaginarios: teoría, metodología y aplicaciones. Santiago de Compostela: CAPA, 6.

DAVIS, W. 1986. Comments on Nathalie Franklin, «Stochastic vs. Emblemic: an archaeologically useful method for the analysis of style in australian rock art». Rock Art Research 3: 124-25.

DELGADO, L. 1988. Los componentes estéticos de la práctica social. Notas para el estudio del arte prehispánico. Boletín de Antropología Americana, 18: 33-48. Instituto panamericano de Geografía e Historia. Caracas.

DELGADO, L. 1988. Seis ensayos sobre estética prehispánica en Venezuela. Caracas: Estudios, monografías y ensayos. Biblioteca de la Academia Nacional de la Historia: 133.

DELNEUF, M. 1991. Un champ particulier de l'experimentation en cerámique: les ateliers de poterie traditionnelle du Nord-Cameroun. En 25 ans d'études technologiques en préhistoire. Bilan et perspectives: 65-82. XI Rencontres Internationales d'Archéologie et d'Histoire d'Antibes. Actes des rencontres 1990, Ville d'Antibes: Éditions APDCA Juan-les-Pins: 397.

«CUADERNOS DE ESTUDIOS GALLEGOS», Tomo XLVIII, Fascículo 114, Santiago 2001. 
EVANS, J. y MILLET, M. 1992. Residuality revisited. Oxford Journal of Archaeology, 11: 225-40. Oxford.

FOUCAULT, M. 1980. La microfisica del poder. Madrid: La Piqueta.

FOUCAULT, M. 1981. Diálogos sobre el poder. Madrid: Alianza Editorial.

FOUCAULT, M. 1984. Vigilar y castigar. Madrid: Siglo XXI (1 $1^{\text {st }}$ ed. 1966. París).

GARCÍA ALÉN, L. 1984. Funcionalidad y sentido estético en las vasijas de los alfareros-campesinos de Galicia. In Jornadas Cientificas Sobre Cerámica y Vidrio (Galicia, 1-3 junio 1981), Santiago de Compostela: Universidad de Santiago de Compostela: 57-73.

GOSSELAIN, O. P. 1992. Technology and style: potters and pottery among Bafia of Cameroon. MAN (New Studies), 27: 559-86.

GRUBER, J. 1986. Archaeology, history and culture. In d. Meltzer, J. Sabloff y D. Fowler (eds.): American archaeology, past and future. Washington D.C.: Smithsonian Institution: 163-86.

HILL, J. D. 1995. Ritual and rubbish in the iron age of Wessex: a study on the formation of a specific archaeological record. Oxford: Tempus Reparatum, BAR, British Series 242.

HODDER, I. 1988. Interpretación en arqueología. Corrientes actuales. Barcelona: Ed. Crítica. ( $1^{\text {st }}$ ed. 1986. Cambridge).

JULIEU, M. 1992. La technologie et la typologie. In J. Garanger (dir.). La préhistoire dans le monde. Nouvelle éditión de la préhistoire d'André Leroi-Gourhan: 162-93. París: Nouvelle Clio.

LAKOFF, G. 1987. Women, Fire and Dangerous Things. Chicago.

LEMONNIER, P. 1983. L'étude des systèmes techniques, une urgence en technologie culturelle. Téchniques culturelles 1: 11-26. 
LEMONNIER, P. 1986. The study of material culture today: toward an anthropoloy of technical systems. Journal of Anthropological Archaeology 5: 147-86.

LEMONNIER, P. 1991a. De la culture matérielle á la culture? Ethnologie des techniques et préhistoire. En 25 ans d'études technologiques en préhistoire. Bilan et perspectives: 15-20. XI Rencontres Internationales d'Archéologie et d'Histoire d'Antibes. Actes des rencontres 1990, Ville d'Antibes: Éditions APDCA Juan-les-Pins: 397.

LEMONNIER, P. 1991b. Technological choices. Transformation in material cultures since the Neolithic. Londres: Ed. Routledge.

LÉROI-GOURHAN, A. 1965. Préhistoire de l'art occidental. (1 $1^{\text {st }}$ ed. Paris).

LÉVI- STRAUSS, C. 1986. La alfarera celosa. Barcelona: Paidós Studio/ Básica: 213. ( $1^{\text {st }}$ ed. 1985. París).

LÉVI- STRAUSS, C. 1987. Antropología estructural. Barcelona: Ed. Paidós: 428. ( $1^{\text {st }}$ ed. 1974. Buenos Aires).

MALTBY, M. 1985. Patterns in faunal assemblage variability . In G. Barker y C. Gamble (eds.): Beyond domestication in prehistory Europe: 3374. London: Routledge.

MARTINÓN-TORRES, M. 2002. Châine opératoire: the concept and its applications within the study of technology. Gallaecia, 21: 29-43. Santiago de Compostela.

MÉNDEZ FERNÁNDEZ, F. 1994. La domesticación del paisaje durante la Edad del Bronce gallego. Trabajos de Prehistoria, 51, $\mathrm{n}^{\circ}$ 1: 77-94. Madrid.

PERLÉS, C. 1991. Économie des matérie premiére et économie du débitage: deux conception opposeés?. En 25 ans d'études technologiques en préhistoire. Bilan et perspectives: 35-46. XI Rencontres Internatio- 
nales d'Archéologie et d'Histoire d'Antibes. Actes des rencontres 1990, Ville d'Antibes: Éditions APDCA Juan-les-Pins.

PRIETO MARTÍNEZ, P. 1993. Aproximación al análisis formal de la cerámica de la Edad del Bronce en Galicia. Trabajo de Investigación, inédito. Santiago de Compostela: Departamento de Historia I, Facultade de Xeografía e Historia, Universidade de Santiago de Compostela.

PRIETO MARTÍNEZ, P. 1995. Definición de un sistema metodológico para el estudio de la cerámica de la Edad del Bronce en Galicia: La tradición campaniforme del yacimiento de A Lagoa (Toques, A Coruña). In Actas del XXII Congreso Nacional de Arqueología (Vigo, 1993); vol. II: 17-24. Vigo: Concello de Vigo, Consellería de Cultura e Xuventude, Xunta de Galicia.

PRIETO MARTÍNEZ, P. 1996. Estudio de la cerámica del segundo milenio a.C. de la Sierra del Bocelo y el Occidente gallego. Tesis de licenciatura, Inédita. Santiago de Compostela: Facultade de Xeografía e Historia, Departamento de Historia I, Universidade de Santiago de Compostela.

PRIETO MARTÍNEZ, P. 1998. Forma, estilo y contexto en la cultura material de la Edad del Bronce gallega: cerámica campaniforme y cerámica no decorada. Tesis doctoral editada en CD-Rom (1999). Santiago de Compostela: Facultade de Xeografía e Historia, Departamento de Historia I, Universidade de Santiago de Compostela.

PRIETO MARTÍNEZ, P. 1999. Caracterización del estilo cerámico de la Edad del Bronce en Galicia: cerámica campaniforme y cerámica no decorada. Complutum, 10: 71-90.

PRIETO MARTÍNEZ, M. P. 2002a. A formal characterisation of ceramics in Galician Bell-Beaker contexts. Studia Vasorum, 1 (www.studiavasorum.ro/ppm.htm).

PRIETO MARTÍNEZ, M. P. 2002b (in press). Ceramic style in Neolithic societies in Galicia (NW Iberian Peninsula). Similarities and differ- 
ences in patterns of formal regularity. In Acts of the XIVth Congress of the U.I.S.P.P. Liège, Belgique, 2-8 septembre 2001, Section 9: Néolithique au Proche Orient et en Europe / Neolithic in the Near East and Europe), Sessions générales / General Sessions. Oxford: Archaeopress.

PRIETO MARTÍNEZ, M. P. 2002c (in press). Ceramic style in Bronze Age societies in Galicia (NW Iberian Peninsula). Similarities and differences in patterns of formal regularity. In Acts of the XIVth Congress of the U.I.S.P.P. Liège, Belgique, 2-8 septembre 2001, Section: 11. Age du Bronze en Europe et en Méditerranée / Bronze Age in Europe and the Mediterranean, Sessions générales / General Sessions. Oxford: Archaeopress.

RIVERA DORADO, M. 1990. El reflejo de la memoria. Notas sobre arte y arqueología. Revista Española de Antropología Americana, 20: 1933. Madrid.

SACKETT, J. R. 1983. From de Mortillet to Bordes: a french paleolithic research. In G. Daniel (ed.): Towards a history of archaeology :59112. London: Thames and Huston.

SACKETT, J. R. 1990. Style and ethnicity in archaeology: a case for isochrestism. In M.W. Conkey y C.A.Hastorf (eds.): The uses of style in archaeology: 32-43. Cambridge: Cambridge University Press.

SCHIFFER, M. 1976. Behavioral archaeology. London/New York: Academic Press.

SHANKS, M. y TILLEY, C. 1987. Reconstructing in archaeology. Cambridge: Cambridge University Press.

VARELA TORRECILLA, C. 1990. La producción alfarera artesanal del occidente de la Península del Yucatán: un ejemplo de cambio cultural. Revista Española de Antropología Americana, $\mathrm{n}^{\circ} 20$. Ed. Universidad Complutense: 183-220. Madrid. 